\title{
Instabilities in yielding
}

\author{
G VENKATARAMAN \\ Reactor Research Centre, Kalpakkam 603 102, India
}

\begin{abstract}
This paper addresses itself to instabilities observed during tensile testing, and complements the papers of Rodriguez and Ananthakrishna presented at this Meeting. The work of Caglioti et al on the elastic to plastic transformation is first reviewed. The work of Kubin $e t$ al on the serrated (repeated) yielding observed at liquid helium temperatures is then discussed in brief. Finally, our own work relying on electronic simulation is described. We conclude with some brief remarks on a few important questions that merit attention in the future.
\end{abstract}

Keywords. Tensile testing; yielding; instabilities; electronic simulation.

\section{Introduction}

Yielding observed during a tensile test is being discussed by several speakers at this Meeting. While metallurgists are by and large interested in the deformation mechanisms, for physicists the nonlinearities and the instabilities arising thereof are perhaps attractive avenues for investigation. Dr Rodriguez will present the metallurgist's point of view while Dr Ananthakrishna will discuss how he views the phenomena as a physicist. My talk will deal with some work on the subject that is pertinent to the present Meeting but not touched upon by them.

\section{Elastic to plastic transition}

The work done by Prof. Caglioti and coworkers (Boffi et al 1980) who address themselves to the instability associated with the elastic to plastic transition is first reviewed. They adopt a hydrodynamic approach, and their basic idea is as follows. One starts with the Helmholtz free energy $F$ expressed as a function of the temperature $T$ and strain $\varepsilon$, since these are quantities which can be measured in real time during an experiment. $F(T, \varepsilon)$ is expanded in a series in $\varepsilon$ and $\theta=\left(T-T_{0}\right) / T_{0}, F\left(T_{0}, 0\right)$ being the free energy of the undeformed solid at temperature $T_{0}$, assuming that a uniaxial tension is applied. By differentiating $F(T, \varepsilon)$ appropriately with respect to the state variables, expressions are deduced for the entropy $S$ and the equilibrium stress $\sigma_{e}$. These are substituted in the space- and time-dependent partial differential equations representing entropy and momentum balance. This is the starting-point for the subsequent stability analysis, the procedure up to this point being identical to what is normally done in hydrodynamics.

We are interested in the question of stability as the material deforms through the elastic region. Let us suppose that the applied (constant) strain rate is $\dot{\varepsilon}_{a}$. To investigate the stability of a particular state $\varepsilon_{a}\left(=\dot{\varepsilon}_{a} t, t\right.$ being the elapsed time) and temperature $\theta$, 


\section{MODE STABILITY}
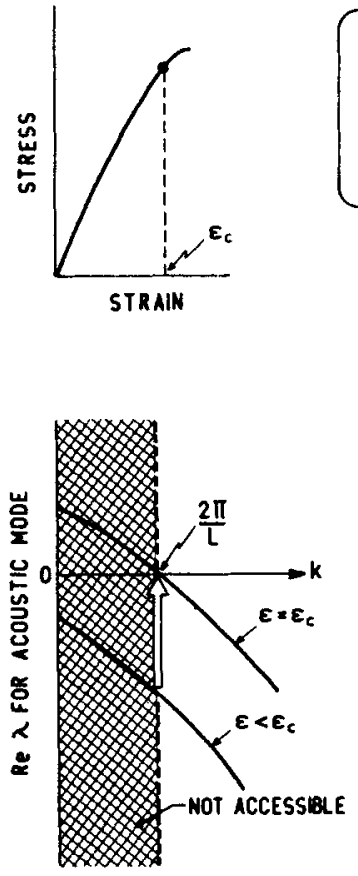

\section{EXCITATION WAVE}

$\sim \exp (i k x+\lambda t)$

FOR STABILITY

$\operatorname{Re} \lambda(k, \varepsilon, \dot{\varepsilon})<0$

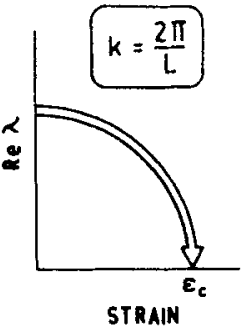

Figure 1. Instability of acoustic mode as $\varepsilon \rightarrow \varepsilon_{\varepsilon}$. Owing to finite size of the specimen, the shaded region is not accessible.

one imposes small fluctuations $\delta \varepsilon$ and $\delta \theta$ around the reference state. Linearization yields a set of three coupled first-order equations for the quantities $\delta \theta,\left(\delta \dot{\varepsilon} / \varepsilon_{a}\right)$ and $\delta \varepsilon$, on which the standard normal-mode analysis may be performed. (In fluid dynamics, one would obtain the Rayleigh and Brillouin modes in this manner.) For a given mode to be stable, it is necessary that $\operatorname{Re} \lambda(k)<0$, where $\lambda$ is the corresponding eigenfrequency and $k$ is the wavevector. It turns out that all the modes are stable as long as $\varepsilon_{a}$ is smaller than a critical strain $\varepsilon_{c}$. At the critical strain, the acoustic mode with the longest wavelength (i.e., $k \approx 2 \pi / L$ where $L$ is the length of the sample) becomes unstable (figure 1 ). There is an obvious analogy with phase transitions; Boffi et al (1980) have indeed drawn a parallel with the nonequilibrium transition in the laser. As yet there is only partial experimental confirmation of these ideas, but I understand (Bottani, private communication) that efforts are in progress to monitor the transition more completely by measuring the associated temperature fluctuations.

\section{Repeated yielding}

Let us consider next the instability aspects of serrated yielding. Dr Rodriguez in his presentation offers a comprehensive survey of the various mechanisms responsible for repeated yield drop. Dr Ananthakrishna considers one of these models, namely, that due to Cottrell, and analyzes how instabilities can arise. I shall complete the picture by discussing the work of Kubin et al and some of our own work. 


\subsection{Low-temperature yielding}

Kubin and coworkers (Estrin and Kubin 1981; Kubin et al 1982, 1983) are concerned with repeated yield drops observed at liquid helium temperatures. These arise due to thermomechanical instabilities for which a model is proposed. The stability analysis is rather similar to that carried out by Boffi et al (1980) (the applied stress and the temperature are now chosen as the state variables characterizing the material undergoing deformation). These two variables are coupled through two nonlinear differential equations, the mechanical equation and the equation describing heat removal, in which the experimental conditions are suitably introduced. Linearization, stability analysis, etc., are then performed as usual, and contact is made with elementary bifurcation theory (Haken 1977).

The parameters that enter into the thermomechanical model of Kubin and coworkers (Estrin and Kubin 1981; Kubin et al 1982, 1983) are: the specimen dimensions, the applied strain rate, the combined elastic modulus of the specimen and the machine, the specific heat of the specimen and the coefficient of heat transfer. These parameters are combined into three dimensionless quantities $\Sigma_{a}$ (the reduced elastic slope of the straining curve), $\alpha$ (the reduced activation volume) and $v$ (representing, apart from a factor, the ratio of the elastic and thermal energies). These are the control parameters. Figure 2 shows the $\left(v / \alpha, \dot{\Sigma}_{a} \alpha\right)$ plane in control parameter space. The different regions represent qualitatively different kinds of mechanical behaviour, the nature of which is amplified in figure 3 . The types of trajectories in the phase space $(\sigma, \theta)$ associated with the various regions in figure 2 are sketched in figure $3 a$, and the corresponding yield curves are shown in figure $3 \mathrm{~b}$. While one has an asymptotic steady state in region II, one obtains serrations in region III. The crossing of the II-III boundary corresponds to a hard-mode instability. (A similar instability is discussed by Ananthakrishna with reference to his model.) To sum up, the work of Kubin et al demonstrates that it is possible to predict whether plastic deformation at low (cryogenic) temperatures will be smooth or jerky, depending on the values of a specified set of mechanical, geometric and thermal parameters, by using bifurcation theory and stability analysis.

\subsection{Electronic simulation}

I now turn to the work on serrated yielding which Neelakantan and myself (1983) have been engaged in recently. Our work was triggered by a fascinating paper by Franck

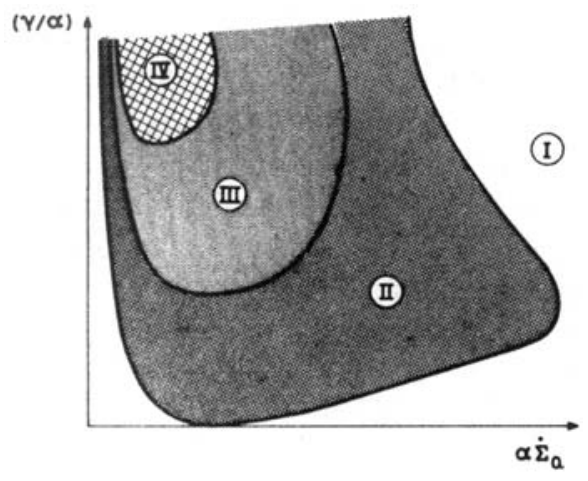

Figure 2. Regions of different types of mechanical behaviour in the parameter plane $\left(\Sigma_{\alpha} \alpha, v / \alpha\right)$ (Kubin et al 1983). 

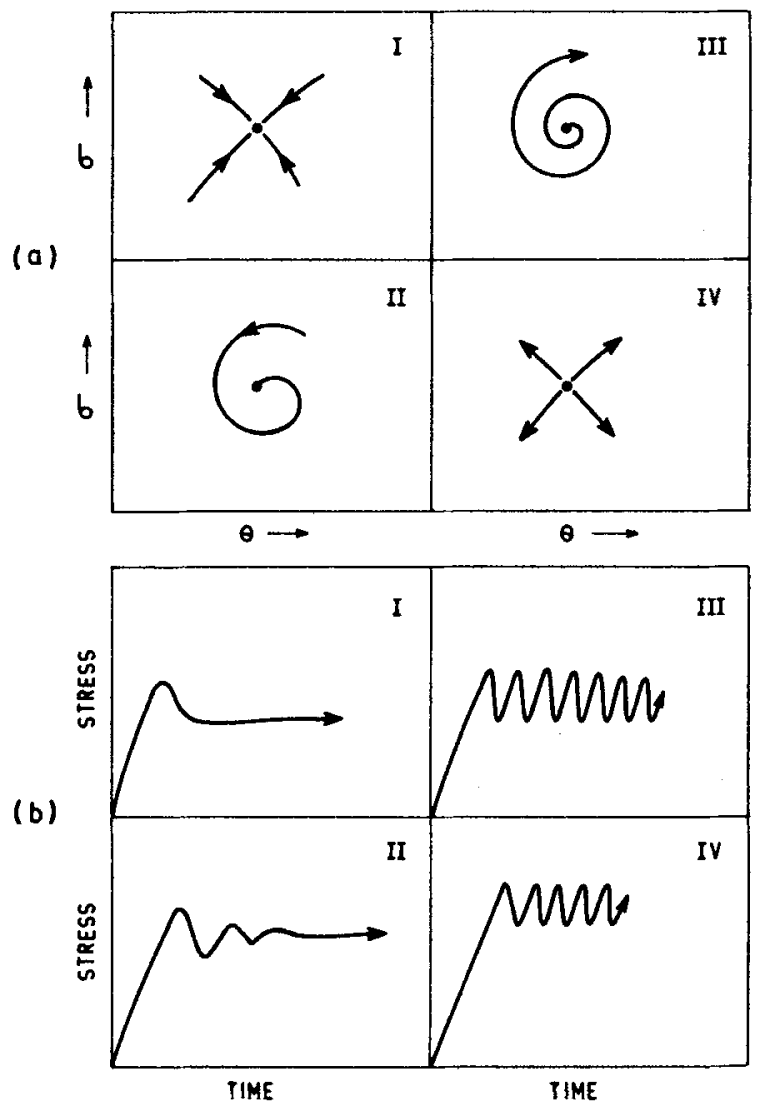

Figure 3. a. Phase space plots corresponding to the different regions shown in figure 2 . b. Stress $v$ time curves corresponding to the different regions of figure 2 (Kubin et al 1983).

(1974) which gives an overview of feedback oscillations occurring in widely different chemical and biological systems. According to Franck, these diverse systems exhibit very similar behaviour because they share an underlying "negative resistance" feature. In serrated yielding, it is well known that at least for one class of models, viz., the Cottrell-Bilby model (1949) (see also the paper by Rodriguez at this Meeting), there is such a negative resistance feature. We (Neelakantan and Venkataraman 1983) therefore explored how some of the observed features of repeated yielding could be understood along the lines indicated by Franck. It was most convenient to map the problem onto an equivalent electronic problem, and essentially carry out an analog simulation.

Skipping the details, the essential idea underlying our work may be understood by referring to figure 4 . Shown here is a typical negative resistance characteristic, the detailed nature of which is dependent (in the physical problem under consideration) on parameters such as the dislocation density, temperature, etc. The applied strain rate prescribes the load line; the behaviour of the specimen during the test depends on where the load line cuts the characteristic curve. If the intersection is in the negative resistance region, then there is instability and oscillations (in stress) ensue.

Now, in contrast to the situation obtained usually in an electronic device, the characteristic curve itself evolves during the test for a specimen undergoing plastic 

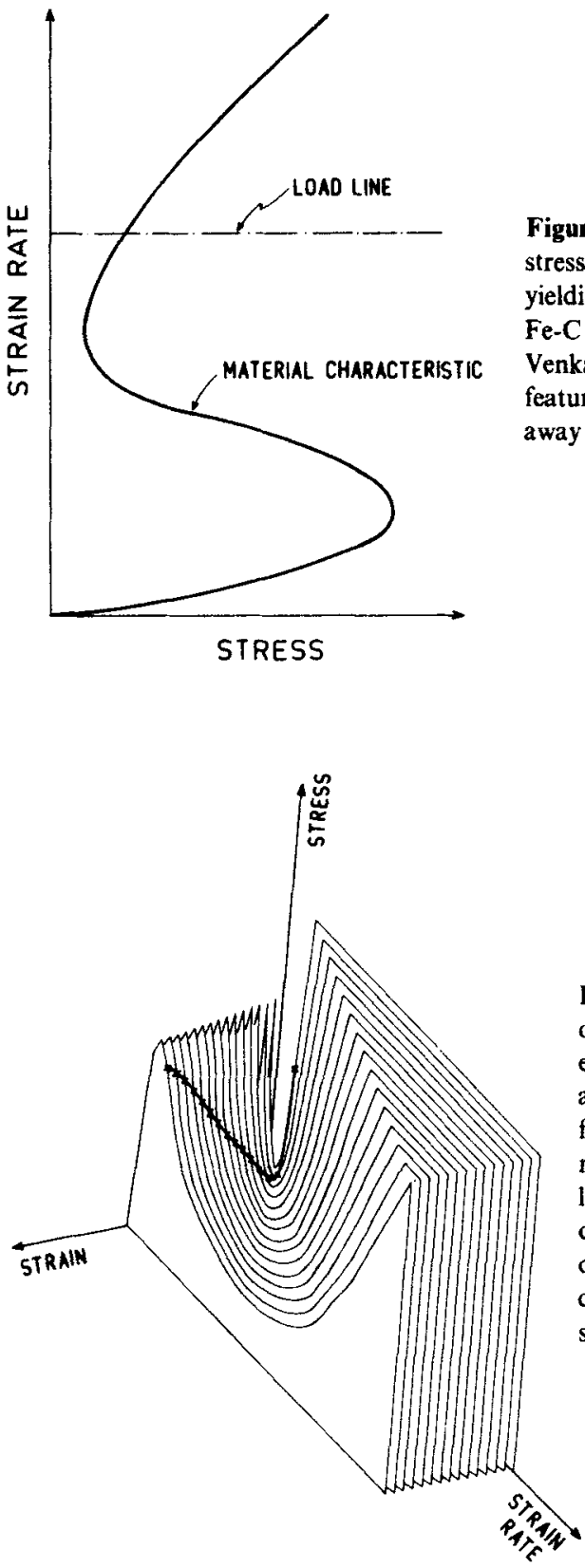

Figure 4. Schematic plot of the strain rate $v s$ stress curve for a material exhibiting serrated yielding. (For examples of such curves in the Fe-C system, see Neelakantan and Venkataraman 1983). The negative resistance feature arises due to dislocations breaking away from their atmospheres.
Figure 5. Perspective plot of a family of characteristic curves, simulating the evolution during an actual test. The valley associated with the negative resistance feature is seen to widen as the test progresses. The trajectory obtained by the load line method is also partially indicated. Serrations occur during the test only when the load line intercepts the characteristic in a region of negative slope.

yields. Thus, at a fixed temperature, and for the same material, the intersection of the load line and the characteristic may occur in a region of positive slope at one instant and in a region of negative slope at the next. The general form of the characteristic also suggests why serrations occur only in a certain region of the test curve. Figure 5 shows a schematic plot of a family of evolving characteristic curves for a material at a particular temperature. The trajectory resulting from the load line is also indicated, and one can see that instability can occur in certain ranges of the strain and not in others. 
Our approach demonstrates that numerous aspects of tensile test curves observed in practice are readily reproduced and studied under convenient, controlled conditions in a suitably constructed analog device. One can begin to understand some of the observed trends in depth. For example, it is known that at a given temperature, serrations are absent both at very high and low strain rates; likewise, at a given strain rate, serrations appear only in a certain temperature band. There is therefore a welldefined region in the $\dot{\varepsilon}-T$ plane within which serrations occur. Our study shows that such a bounded region is indeed to be expected.

\section{Conclusions}

One common feature of all the models cited is that they tacitly assume the medium to respond homogeneously during repeated yielding. There are, however, reasons to believe that the deformation is in fact spatially inhomogeneous. This aspect has not received the attention it deserves in theoretical studies of the phenomenon, and one hopes that in the years to come this will be set right.

Another relatively neglected aspect is the important role played by noise: a considerable amount of irregularity exists in the observed serrations. Ananthakrishna discusses the effects of fluctuations to some extent in his paper. In our work, too, some thought has been given to the matter. Our preliminary finding is that when noise is included, the simulated tensile test curves begin to resemble real-life curves such as, e.g., those found in Hall (1970). This work is still in progress. One's experience with the study of fluctuations in other areas of physics suggests that here, too, such an analysis would be a fruitful avenue of investigation.

\section{References}

Boffi S, Bottani C E, Caglioti G and Ossi P M 1980 Z. Phys. B39 135

Cottrell A H and Bilby B A 1949 Proc. Phys. Soc. 16249

Estrin Y and Kubin L P 1981 in Deformation of polycrystals Proc. 2nd Ris $\phi$ Int. Symp. Metal. Mat. Sci., (eds) N Hansen, A Horserrell, T Leffers and $H$ Lilholt p. 179

Franck U C 1974 in Physical chemistry of oscillatory phenomena Symp. Faraday Soc. No. 9, p. 137

Haken H 1977 Synergetics (Berlin: Springer)

Hall E O 1970 Yield point phenomena in metals and alloys (New York: Plenum)

Kubin L P, Spiesser Ph and Estrin Y 1982 Acta Me:zll. 30385

Kubin L P, Estrin Y and Spiesser Ph 1983 preprint, Low temperature plastic deformation of metals and bifurcation theory (to appear in Res. Mechanica)

Neelakantan K and Venkataraman G 1983 Acta Metall. 3177

\section{Discussion}

G Srinivasan: The introduction of noise seems to produce bands. Is it that you are seeing some kind of a beat phenomenon?

G Venkataraman: We have not explored all the possibilities to say something at this stage.

R Ramaswamy: The kind of bunching phenomenon that occurs does have a practical 
analogue. This is the phenomenon of intermittency-shortly before the onset of a truly turbulent phase, long range periodicity appears in bursts.

K R Rao: Referring to Caglioti et als work, in the yield instability region, the soft mode may go to zero giving rise to an incommensurate phase reciprocal lattice point. Has this been experimentally observed?

Venkataraman: It has not been observed. This is only a model. 